\title{
Collaborative acute medical postgraduate-undergraduate simulation: a new multi-modal transition programme for final-year medical students
}

\author{
Authors: Sanna Khawaja, Aditya Bhalla, Viral Patel, Shairana Naleem, Abiola Suleiman, Nira Rangasalen and \\ Anthony Kenny
}

\begin{abstract}
Aims
The collaborative acute medical postgraduate-undergraduate simulation (CAMPUS) initiative is a pilot endeavour to optimise the undergraduate-postgraduate interface. It is grounded in the dynamic final-year undergraduate acute medicine (AM) placement at Kings College Hospital, Denmark Hill, aiming to immerse students in the realities of front-line medicine and professionalism, in keeping with General Medical Council (GMC) good medical practice.

Central to this innovative programme is the unique approach of collaborative learning with final-year medical students alongside practising front-line AM nurses in active clinical risk management. Key educational strategies encompass case-based learning, team building exercises and high-fidelity simulation-based (SB) learning scenarios with dedicated ethical and communication components. Delegates engage in active reflection on human factors, professionalism, evidence-based best practice, non-technical skills and consideration of a holistic approach to the mental-physical interface.
\end{abstract}

\section{Methods}

An initial learning-needs assessment was carried out using a survey sent to all candidates in advance. The course was then tailored to meet training needs highlighted by students to address gaps potentially impacting the transition from student to clinician.

The programme was delivered to two band 6 AM nurses and 16 medical students, facilitated by front-line consultants, registrars and nursing technical staff. It consisted of team building, case reviews, high-fidelity simulation of complex emergencies and communication scenarios. All components were followed by a debrief providing the latest evidence-based guidance to consolidate learning, as well as a safe environment to reflect on human factors. Throughout the course, the learning points were linked back to the GMC's guidance on good medical practice. Candidates completed a post-course survey to assess the impact

Author: Acute Medicine, King's College Hospital, London, UK

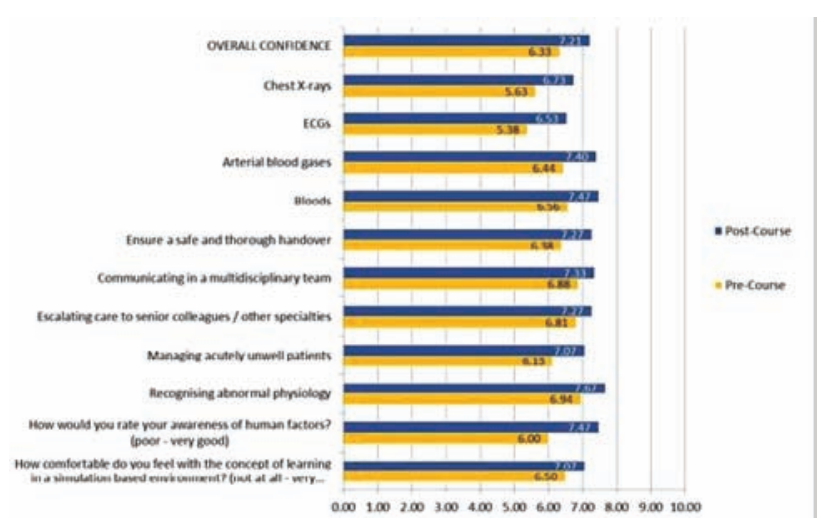

Fig 1. Mean confidence ratings of candidates.

and utility of the CAMPUS pilot as a training tool in helping prepare candidates for life as foundation year one doctor.

Results

Qualitative feedback was obtained in the form of pre- and postcourse Likert surveys. This consisted of confidence ratings and freetext boxes. Candidates reported variable degrees of experience with previous SB teaching. Fig 1 summarises the results of the surveys; confidence ratings in various domains, ranging from clinical management, interpretation of investigations and non-technical skills that were gathered. The post-course survey demonstrated improved mean confidence in every criterion assessed. The overall confidence rating improved from $63.03 \%$ to $72.06 \%$.

\section{Conclusion}

The results demonstrate that the CAMPUS pilot succeeded in increasing candidates' confidence in all assessed domains. The largest improvement in reported confidence levels was seen in awareness of human factors, with accompanying qualitative feedback appreciating the 'safe environment' to reflect. Qualitative feedback was also vital in helping to identify the components of 
the course that were most appreciated, ranging from 'assessing a patient with the ABCD (approach)' to the logistical planning such as 'video set-up was amazing'. Most candidates reported a strong desire for increased SB teaching, eg 'sessions are generally extremely useful and it would be brilliant if more could be made available to us'.

A limitation of this study is that to maximise the learning opportunities the number of candidates was limited, hence producing a small sample size. Outcomes were enriched through detailed qualitative feedback. Going forward our future vision is to embed CAMPUS as part of multidisciplinary continuous learning culture within King's AM, as well as supporting the successful transition of final-year medical students from the protected undergraduate environment to the high intensity AM arena. This area of training is currently not standardised across the country and is due to be further addressed by the GMC with the introduction of the universal licensing exam in 2022. Medical and foundation schools nationally would benefit from considering similar strategies for alignment with this important step.

\section{Conflict of interest statement}

There are no known conflicts of interest to declare. 